XX.

Aus der Universitäts-Ohrenklinik zu Berlin.

(Direktor: Geh. Med.-Rat Prof. Dr. P a s s ow.)

\title{
Kritische Bemerkungen über das Empyem des Saccus endolymphaticus und die Bedeutung des Aquaeductus vestibuli als Infektionsweg.
}

Von

Dr. Oskar Wagener, Assistenten der Klinik.

In einer ausführlichen Arbeit hat Boesch') auf die Bedeutung hingewiesen, die dem Aquaeductus vestibuli zukommen soll, wenn entzündliche Prozesse vom erkrankten Labyrinth auf das Schädelinnere übergreifen. Er fand in der Literatur 65 Fälle, in denen der Infektionsweg vom inneren $\mathrm{Ohr}$ nach dem Schädelinneren ausdrïeklich angegeben ist; in 21, also $1 / 3$ dieser Fälle wird der Aquaeductus vestibuli als Infektionsweg bezeichnet. Hinsber ${ }^{2}{ }^{2}$ ) dagegen konnte unter 43 Fällen nur $8=18,6$ Proz. finden, bei denen dieser Infektionsweg angenommen ist. Und Politzer ${ }^{3}$ ) sagt sogar in einer soeben ersohienenen Arbeit, daß zwar nach Beobachtungen anderer Autoren eine Eiterung im Vorhofe anf dem Wege des Aquaeductus vestibuli zu einem Empyem des Intraduralsackes der Vorhofswasserleitung führen könnte, daß diese Komplikation ihm aber eine sehr seltene zu sein schiene.

1) Boesch, Der Aquaeductus restibuli als Infekionsweg. Zeitschrift für Ohrenheilkunde. 1905. 50. Bd. S. 337.

2) Hinsberg, Über Labyrintheiterungen. Zeitschrift für Ohrenheilkunde. 40. Bd. 1902. S. 117.

3) Politzer, Labyrinthbefunde bei chronischen Mittelohreiterungen. Archiv für Obrenheilkunde. 1905. 65. Bd. S. 161. 
Friedrich 1) hält die Entstehung eines Saceusempyems nur bei ausgebreiteter Labyrintheiterung für möglich, mißt ihm aber nur die Bedeutung eines extraduralen Abszesses bei. Im Gegensatz zu den von anderer Seite makroskopisch konstatierten Obduktions- und Operationsbefunden hat er in mehreren Fällen bei starker entzändlicher Neubildung in Schnecke und Vorhof und Erfullung des Aquaeductus cochleae mit Eiter durch mikroskopische Untersuchung das Intaktsein des Aquaeductus vestibuli nachweisen können. Die Zahl der makroskopisch diagnostizierten Fälle scheint ihm im Widerspruch zu den mikroskopisch sichergestellten Fällen zu stehen.

Der Liebenswïrdigkeit meines früheren Chefs, des Herrn Prof. Zinn, verdanke ich nun einen Fall, der makroskopisch den typischen Eindruck eines Saccusempyems machte, der sich aber mikroskopisch als einfacher extraduraler Abszeß erwies. Dieser uberraschende Befund veranlaßte mich, die bisher beschriebenen Fälle von Saccusempyem einer genauen Durchsicht zu unterziehen.

Aus der Krankheitsgeschichte des Falles sei nur folgendes mitgeteilt:

Am 14. November 1903 wurde der 28 jäbrige Klempner E. W. auf die innere Station des Krankenhauses Bethanien in Berlin eingeliefert. Der Patient war benommen und hochgradig schwerbörig, die Aufnahme der Anamnese stieb daher auf Schwierigkeiten. Es lieb sich nur feststellen, daB Patient seit ungefäbr 3 Jahren ohrenlejdend und schwerhörig war, und seit einigen Tagen Kopfschmerzen hatte. Beiderseits bestand eine stinkende Ohreiterung. 2 Tage nach der Aufnahme starb der Kranke unter meningitischen Symptomen.

Die Sektion, die erst 49 Stunden nach dem Tode gemacht werden konnte, ergab als Todesursache einen rechtsseitigen KleinhirnabszeB. Die ubrigen Organe boten keinen besonderen patbologischen Befund.

Der AbszeB im rechten Kleinhirn war etwa walnußgroß, ziemlich scharf gegen die übrige Hirnsubstanz abgesetzt und enthielt schmutzig grünlichen Fiter. An der Hinterseite des rechten Felsenbeins war der Abszel mit den weichen Hirnhäuten und der Dura mater an einer runden, ungefähr $1,5 \mathrm{~cm}$ im Durchmesser haltenden Stelle ziemlich fest verwachsen; beim Herausnebmen des Kleinhirns rils diese Stelle ein, und es entleerte sich hier aus dem Abszeb viel Eiter.

Die Verwachsungsstelle des Kleinhirn.s an dem Sitze des Abszesses mit der Hinterseite des Felsenbeins war, wie schon erwähnt, fast kreisrund und befand sich an der Stelle, wo der Saccus endolymphaticus zu liegen pflegt, etwas lateral von der Mitte zwischen Porus acusticus internos und Sinus, diesem bis auf ca. $0,5 \mathrm{~cm}$ genăhert. Mit leicht ausgezacktem, etwas aufgerichtetem Rande setzte sich dieser Defekt, dessen Grund mit wenig bräunlichen, fest baftenden Massen bedeckt war, scharf gegen die übrige glänzende Dura ab. Über den Zustand des Knochens an der betreffenden Stelle war nichts Bestimmtes zu sagen.

1) Friedrich, Die Eiterungen des Ohrlabyrinths. Wiesbaden: 1905.. Bergmann. S. 53 ff. 
Kritische Bemerkungen über das Empyem des Saccus endolymph. usw. 275

Bei diesem makroskopischen Befunde schien es sicher, dab es sich um einen Abszeb des Saccus endolymphaticus handelte, von dem aus der Hirnabszeh entstanden war.

Auch die mikroskopische Untersuchung des Felsenbeins schien zuerst diese Annahme za bestätigen; erst bei systematiseher Verfolgung des Aquaeductus vestibuli zeigte es sich, daß der Sacous endolymphaticus nioht erkrankt war, sondern daß er als feiner Spalt neben dem Abszeb lag, der dureh Zerstörung des Knochens an einer ganz kleinen Stelle am Grunde desselben entstanden war.

Das ganze Labyrinth, auch der Aquaeductus vestibuli, war frei von pathologischen Veränderungen. Einzelheiten, z. B. am Cortischen Organ, ließen sich allerdings nicht mehr erkennen, was wohl darauf zurlickzufubren ist, daß die Sektion erst 48 Stunden nach dem Tode gemacht werden konnte. Im übrigen $\mathrm{Knoohen}$ aber zeigte sich eine starke Durehsetzung mit Eiter, und es hatte sich an der Hinterseite des Felsenbeins dicht neben dem Saceus endolymphaticus eine feine cariöse Stelle gebildet, die zur Bildung eines extraduralen Abszesses an dieser Stelle geführt hatte.

Offenbar war der Vorgang folgender gewesen: Auf der Höhe der Kuppe des Abszesses war die Dura nach Verklebung mit dem Kleinhirn nekrotiseh geworden, und es fanden sich jetat nur noch die abgehobenen Randteile, die aber deutlich auf dem Durehschnitte eine Spaltung in zwei Blätter erkennen ließen, von denen jedes etwa die Hälfte der Dicke der Dura mater besaß. Gerade diese Aufsplitterung der Dura hatte auch noch im mikroskopischen Bilde zuerst zu der Annahme geführt, daß es sich wirklich um den vereiterten Saceus endolymphaticus handelte. Dieser lag aber völlig gesund neben dem Abszeß, jedoch so, daß die eitrige Infiltration der Dura noeh etwas den Rand des Saccus umfaßte. Dieser aber lag auffallend weit von dem medialen Rand des Sinus, im mikroskopisehen Präparate gemessen etwa $1,3 \mathrm{~cm}$, gemessen vom Sinus bis zum lateralen Rand des Saccus. In diesen Zwischenraum war der extradurale Abszeß eingelagert. Der Saceus endolymphatiens maß in horizontaler Richtung (das Felsenbein war parallel der oberen Pyramidenkante geschnitten) bis $0,6 \mathrm{~cm}$.

Fassen wir noch einmal das wichtige dieses Falles zusammen:

Ein $A b s z e B$ an der Hinterseite des Felsenbeins imponierte makroskopisoh als geborstenes Sacousempyem. Erst die eingehende mikroskopische Unter- 
suchungergab, daßes sich umeinen einfachen extraduralen AbszeB handelte. Zuerst hatte es den Anschein, als läge in diesem Falle der erste anatomische Beweis vor für die Annahme einiger Autoren ( $\mathrm{K} \ddot{\mathrm{rn}} \mathrm{er}, \mathrm{K} u ̈ \mathrm{mmel}$ ), daß sich ein Empyem des Saceus endolymphaticus bei intaktem Labyrinth entwickeln könne durch Perforation einer Knocheneiterung direkt in den Saceus hinein. Die mikroskopische Untersuchung zerstörte diesen Wahn.

Aus der Literatur habe ich 31 Fälle zusammenstellen können, bei denen entweder nach Angabe der Autoren selbs t ein Saceusempyem auf dem Wege des Aquaeductus vestibuli entstanden war oder die ron anderen Autoren immer wieder als Fälle ron Saceusempyem bezeichnet werden. Von den 21 Fallen, die Boesch (1. c.) in seiner Statistik aufgezählt hat, habe ich 2 Fälle ausgeschaltet: erstens den ron $\mathrm{Okada}^{1}$ ) erwähnten Fall III, der mit dem von Müller ${ }^{2}$ ) beschriebenen identisch ist; sodann den Fall (35) von Hegetsehweiler ${ }^{3}$, wo es sich bei einem 21/2jährigen Kinde um einen ausgedehnten tuberkulösen Prozeb im Schläfenbein, Tuberkulose der Dura mater und Solitärtuberkel des Groß- und Kleinhirns handelte. Es wird zwar angegeben, daß der Kleinhirntuberkel an der Eintrittsstelle des Aquaeductus vestibuli mit dem Knochen verlötet war; da aber auch ein Großhirntuberkel und Miliartuberkulose der Dura vorhanden war, so kann man aus dem Befund, mikroskopisch ist das Felsenbein nicht untersucht, wohl nur den Schluß ziehen, den Hegetsehweiler selbst auch nur zieht, daß wahrscheinlich die Meningitis tuberculosa und die Solitärtuberkel im Gehirn ihren direkten Ausgangspunkt von der Ohrerkrankung genommen haben, nicht aber, daß nun der Aquaeductus vestibuli den Überleitungsweg gebildet habe.

Zwei Fälle von Braunstein ${ }^{4}$ und Heine $e^{5}$ hat Boesch

1) Okada, Diagnose und Therapie des otogenen Kleinhirnabszesses. Klinische Forträge herausgegeb, von Haug III. Bd. 1900. S. 310.

2) Richard Muller, Bericht uber die Ohrenklinik des Geh. Med.Rates Prof. Dr. Trautmann für das Jahr rom 1. April 1896 bis 3L. März 1897. Charité Annalen 22. Bd. 1897. S. 369.

3) Hegetschweiler, Die phthisische Erkrankung des Ohres auf Grund yon 39 Sektionsberichten Bezolds. Wiesbaden 1895.

4) Braunstein, Bedeutung der Lumbalpunktion für die Diagnose intrakranieller Komplikationen der Otitis. Dieses Archiv. LIV. Bd. 1902. S. 7.

5) Heine, Zur Kasuistik otitischer intrakranieller Komplikationen. Dieses Archiv. L. Bd. 1900. S. 252. 
wegen Mangels einer genaueren anatomischen und funktionellen Pruffung des Labyrinths 'nicht mitgezählt; ich stimme hier Boesch völlig bei, wenn er diese Fälle für nicht beweisend hält in der vorliegenden Frage. Aber den Mangel einer genaueren anatomischen Untersuchung teilt der erste mit fast allen anderen Fällen, und beim zweiten liegt schließlich ein Sektionsbericht vor, der den makroskopischen Befund ziemlich genau schildert. Ich möchte wegen Fehlens der funktionellen Prüfung diese beiden Fälle nicht ausscbalten aus der Liste derjenigen, die allenfalls als Fälle von Saccusempyem aufgefaßt werden $\mathrm{k} \not ̈ n n e n$, ohne daß ein Beweis hierfür aber geliefert worden ist.

Aus der inzwischen ersehienenen, von Boeseh noch nicht bertìcksichtigten Literatur habe ich noch 7 Fälle hinzugefügt, und zwar die von Friedrich1) (Fall 15, 19), Politzer') (Fall 2), Grunert und Dallmann ${ }^{3}$ ) (Fall 2,5), Kümmelt), sowie von $\mathrm{Klug} 5$ ) (Fall III). Zähle ich einen gleich zu erwähnenden anderen Fall von $\mathrm{Kümmel}$ sowie den von Muck hinzu, so liegen 31 Fälle in der Literatur vor, bei denen die Entstehung. eines Empyems des Saccus endolymphaticus anf dem Wege des Aquaeductus vestibuli von den Autoren angenommen ist.

Diese 31 Falle sollen in verschiedenen Gruppen besprochen werden.

In einer ersten möchte ich 3 Fälle zusammenstellen, bei denen die Diagnose auf Saccusempyem nicht durch die Sektion bestätigt werden konnte, bei denen nur auf Grund des Operationsbefundes diese Diagnose gestellt wurde. Es sind dies zuerst die beiden Fälle von $\mathrm{Kümmel}{ }^{6}$ ) (Fall 1) und Mueki) (Fall 20), die auch dadurch ror allen anderen eine

1) Friedrich, Die Eiterungen des Obrlabyrinths. Wiesbaden 1905.

2) Politzer, Labyrinthbefunde bei chronischen Mittelohreiterungen. Dieses Archiv. LXV. Bd. 1905. S. 161.

3) Grunert und Dallmann, Jahresbericht über die Tätigkeit der Kgl. Universitäts $O$ brenklinik zu Halle a. S. vom 1. April 1904 bis 31 . Mărz 1905. Dieses Archiv. LXV. Bd. 1905. S. 55.

4) Kümmel, Ein Fall von seröser Meningitis neben Kleinhirnabszeß. Festschrift für Lucae. Berlin 1905. S. 313.

5) $\mathrm{Klug}$, Les suppurations du labyrinthe. Annales des maladies de l'oreille, du larynx du nez et du pharynx. Bd. XXXl. 1905. p. 161.

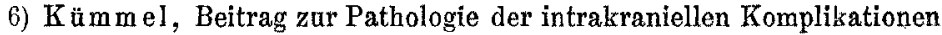
von Ohrerkrankungen. Zeitschr. f. Ohrenheilk. XXVIII. Bd. 1896. S. 254.

7) Muck, Beitrag zur Kenntnis der otitischen Erkrankungen des Hirns der Hirnhäute und der Blutleiter. Zeitschr. f. Ohrenheilk. XXXV. Bd. 1899. S. 218 . 
Sonderstellung einnehmen, daß bei ihnen das Labyrinth nicht erkrankt war, und daß die Autoren die Entstehung des Saceusempyems durch einen direkten Durchbruch einer Knocheneiterung in den Saccus hinein erklären. In dem Fall von Sehenke (Fall 11) war nach der Funktionsprüung wahrseheinlich eine Beteiligung des betreffenden Labyrinthes vorhanden, doch fand sich bei der Operation "wider Erwarten" der hintere vertikale Bogengang gesund. Selbst wenn man die Zuverlässigkeit einer solchen Entscheidung während einer Operation für möglich hält, miißte angenommen werden, daß es sich hier um partielle Labyrintherkrankung gehandelt hat. Als Hauptstütze für die Annahme, daß eine Eiteransammlung zwischen den beiden Durablättern des Sacens endolymphatious dureh Vermittlung des Aquaeductus vestibuli vorhanden gewesen sei, führt Schenke das negative Resultat an beim Suchen eines anderen Weges zum Endoeranium. Und doch heißt es im Operationsbefund: "Hierauf wird der ziemlich weit nach binten gelegene Sinus freigelegt, der Knochen ist in dessen Umgebung nur entzündlich hyperämisch, nirgends Verfärbung oder Eiter. Beim Fortschlagen der Sulcuswand stärzt plötzlich circa ein Kaffeelöffel voll stark stinkenden, diokflüssigen Eiters neben dem Meißel hervor."

Der Knochen war also entzindet, und es ist deswegen die Möglichkeit nicht auszusehließen, daß ein Abszeß an der Hinterseite des Felsenbeins durch den entzündeten Knochen, nicht auf dem Wege des Aquaeductus vestibuli entstanden ist.

Ebensowenig möchte ich auch mit Boesch, Friedrich und anderen den Fall von Kümmel und den Körnerschen, von Muck veröffentlichten Fall als beweiskräftig in der vorliegenden Frage ansehen. In beiden Fällen fanden sich bei der Operation Fisteln, die in die Gegend des Saccus endolymphaticus führten, Muck sagt sogar ,zum Saccus endolymphatieus", dessen dem Knochen anliegende Wand teilweise zerstört war.

Von den übrigen 28 Fällen, die zur Sektionkamen, sind nur 3 Falle mikroskopisch untersucht, bei allen anderen ist makroskopisch die Diagnose auf Saccusempyem gestellt. Bei allen diesen Fällen fand sich makroskopisch das Labyrinth in mehr oder minder ausgedehntem Maße erkrankt.

Den sichersten der in unserer Frage in Betracht kommen-

1) Schenke, Einige Fälle endokranieller Komplikationen akuter und chronischer Mittelohreiterungen. Dieses Archiv. LIII. Bd. 1901. S. 116. 
den Fälle bietet uns wieder Politzer ${ }^{1}$ ) (Fall 2), der bei der mikroskopischen Untersuchung kurz und präcise angiebt, ,der Ductus und Saccus endolymphatious sind mit Eiter gefüllt".

Es ist dies der einzige bis jetzt in der Literatur vorliegende Fall, bei dem einwandfrei das Fortschreiten einer Eiterung vom Labyrinth zwischen die Durablätter beobachtet ist. Denn bei den beiden anderen mikroskopisch untersuchten Fällen von Friedrich') (Fall 15) und Boesch (l. c.) war zwar makroskopisch ein Saccusempyem sehr wahrscheinlich, auch fand sich bei der mikroskopischen Untersuchung Eiter im Ductus endolymphaticus, es ist aber leider nichts davon angegeben, ob und wie mikroskopisch ein $\mathrm{Zu}$ sammenhang zwischen dem makroskopisch diagnostizierten Saccusempyem und dem mikroskopisch festgestellten Eiter im Ductus endolymphaticus bestand. Leider ist auch über den Befund des Knochens an dieser Stelle nichts erwähnt.

Von deu übrigen 24 nur makroskopisch untersuchten Fällen möchte ich wieder sechs zusammenfassen, wo bei der Sektion so ausgedehnte Zerstörungen des Knochens in der Gegend des Saceus vorhanden waren, daß das anatomische Bild hier völlig verwischt ist, so daß sie nicht geeignet sind, als irgendwie beweisende Fälle in unserer Frage herangezogen zu werden, da es sich gerade so gut um extradurale Abszesse hat handeln können.

In dem Fall von $M u ̈ u l l e{ }^{3}$ ) war das Felsenbein um die Mündung des Aquaeductus vestibuli in Ausdehnung einer Linse kariös; in denen von Hein ${ }^{4}$ ) (Fall 60) und $\mathrm{Okada}^{5}$ ) (Fall II) konnte man an dieser Stelle mit Sonden durch Fistelgänge bis ins Mittelohr gelangen Moos und Steinbrügg e ${ }^{6}$ ) sagen, ,an der hinteren Wand der Pyramide in der Umgebung der Apertur des

1) Politzer, Labyrinthbefunde bei chronischen Mittelohreiterungen. Dieses Archiv. LXV. Bd. 1905. S. 161 ,

2) Friedrich, Die Eiterungen des Ohrlabyrinths. Wiesbaden 1905.

3) Richard Müller, Bericht über die Ohrenklinik des Geh. Med.-Rates Prof. Dr. Trautmann für das Jahr vom 1. April 1896 bis 31. Mărz 1897. Charité-Annalen. XXII. Bd. 1897. S. 369.

4) $\mathrm{He}$ in e, Zur Kasuistik otitischer intrakranieller Komplikationen. Dieses Archiv. L. Bd. 1900. S. 252.

5) Okada, Diagnose und Therapie des otogenen Kleinhirnabszesses. Klinische Vortrăge, herausgegeben von $\mathrm{Haug}$. III. Bd. 1900. S. 310.

6) Moos und Stein brügge, Über die histologischen Veränderungen im Knochen und in den Weichteilen des mittleren und inneren Ohres be Karies des Felsenbeins. Zeitschrift f. Ohrenheilk. X. Bd. 1881. S. $8 \pi$ 
Aquaeductus vestibuli war eine tiefere Zerstörung des Knochens sichtbar". Hedinger') (Fall II) fand an dieser Stelle beginnende Nekrose des Felsenbeins in dem Umfang eines Fünfzigpfennigstückes, und Grunert und $S c h u l z e^{2}$ ) konnten sogar in dem das knöcherne Labyrinth umgebenden spongiösen, stark erkrankten Knochen zwischen dem vertikalen Bogengange und dem Porus acusticus internus einen fast $2 \mathrm{~cm}$ langen und $1 \mathrm{~cm}$ breiten Sequester feststellen; es lautet bei ihnen die Diagnose auch nur "ExtraduralabszeB".

Bei 3 Fällen wurde durch die Operation die Dura an der Hinterseite des Felsenbeins in großer Ausdehnung freigelegt, so daß ein Beweis für den Infektionsweg nicht möglich war. Orne Green ${ }^{3}$ ) nimmt zwar für seinen zweiten Fall eine Entstehung: des Kleinhirnabszesses durch Infektion auf dem Wege des Aquaeductus vestibuli an mit Bildung eines Saccusempyems, doch erwähnt er nichts vom Befunde im Vestibulum, in der Schnecke usw. In seinem 3. Falle erklärt er den Kleinhirnabszeb durch Infektion "through the whole labyrinth" entstanden und verzichtet darauf, bei den enormen Zerstörungen des ganzen inneren Ohres einen bestimmten Infektionsweg anzugeben.

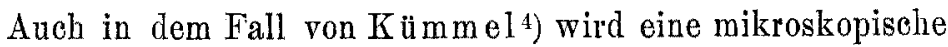
Untersuchung mit Sicherheit den Infektionsweg nicht mehr feststellen können, da die Sektion zeigte, daß hier auch die Gegend des Aquaeductus vestibuli und des Sacous endolymphaticus selbst durch die ausgedehnte Operation vernichtet waren.

Zwei Autoren lassen es selbst fraglich, welchen Weg der Eiter vom Labyrinth zum Sehädelinneren genommen hat; $\mathrm{M} \mathrm{uek}$ ) (Fall 32) nimmt den. Weg durch den Duotus endolymphatious oder

1) Hedinger, Beiträge zur Pathologie und pathologischen Anatomie des Ohres. Zeitschr. f. Ohrenheilk. XIV. Bd. 1885 . S. 47.

2) Grunert und Schulze, Jahresbericht der Kgl. Universitătsohrenklinik zu Halle a. S. 1900/1901. Dieses Archiv. LIV. Bd. 1902. S. 63.

3) Orne Green, Abscesses of the cerebellum from infection through the labyrinth. The American journ. of the medic. sciences. 1899. Bd. CXVII. p. 406 .

4) Kümmel, Ein Fall von seröser Meningitis neben Kleinhirnabszeb. Festschrift für Lucae. Berlín 1905. S. 313.

5) M u ck, Beiträge zur Kenntnis der otitischen Erkrankungen des Hirns, der Hirnhäute und der Blutleiter. Zeitschr. f. Ohrenheilkunde. XXXVII. Bd. 1900. S. 171. 
durch den Hiatus subarcuatus an. Grunert und $\mathrm{Z}$ eron $\mathrm{i}^{1}$ ) (Fall 17) sagen: „Beim Herausnehmen des Kleinhirns riß die an der Rückseite der Pars petrosa des Schläfenbeins angewachsene Dura mit der Abszeßwand ein, dabei entleert sich ein Schuß Eiter. Wo die Verwachsung an der Pars petrosa gesessen hatte, ließsich nachher nioht genau feststellen, wahrscheinlich aber in der Gegend der Mündungdes Aquaeductus vestibuli." Und weiter, "der aufgefundene Kleinhirnabszeb saß in der Nähe der Pyramidenspitze und ist, wie der Sektionsbefund des Sehläfenbeins mit Sicherheit nachwies, durch die vorhanden gewesene Labyrintheiterung induziert worden, wobei jedenfalls der Aquaeductus vestibuli die Rolle des Entzündungsleiters gespielt hat".

In 2 Fällen, dem von Rohden und Kretsehmann ${ }^{2}$ ) (Fall 1) und dem ron Reinhard und Ludewigis) (Fall 6) fand sich etwas Eiter an der Ausmündungsstelle des Aquaeductus vestibuli und in dem ersten Falle in dieser Gegend auch ein "Subduralabszeb". Ob aber der Sacous und der Ductus endolymphaticus hier beteiligt waren, dariber sprechen sich die Verfasser mit keinem Worte aus.

Braunstein ${ }^{4}$ (Fall 2) nimmt an, daß in seinem Fall die eitrige Meningitis wahrschein lich ausgegangen ist rom Aquaeductus vestibuli, $n^{\text {ans }}$ welchem sich beim Ablösen der Dura ein Tropfen Eiter entleerte". Auch Panse ${ }^{5}$ ) (1. Fall) fand nach Entfernung der Dura Eiter an der Ausführungsstelle, die weiter als in der Norm (?) erschien. Er sieht es als sicher an, daß die Eiterung auf diesem Wege, vielleicht durch gleichzeitige Entzündung der Vene sich auf den intrakraniellen Raum fortgesetzt hat.

1) Grunert und Zeroni, Jahresbericht uber die Tätigkeit der Kgl. Universitäts-Ohrenklinik zu Halle a. S. vom 1. April 1898 bis 31. Mărz 1899. Dieses Archiv. IXL. Bd. 1900. S. 97,

2) Rohden und Kretschmann, Bericht über die Tătigkeit der Kgl. Universitäts-Ohrenklinik zu Halle a. S. im Jahre 1886. Dies. Arch. XXV. Bd. 1887. S. 106.

3) Reínhard und Ludewig, Bericht über die Tätigkeit der Königl. Universitäts-Ohrenklinik zu Halle a. S. vom 1. Januar 1887 bis 31 . Mărz 1888. Díeses Archiv. XXVII. Bd. 1889. S. 201.

4) Braunstein, Bedeutung der Lumbalpunktion für die Diagnose intrakranieller Komplikationen der Otitis. Dieses Archiv. LIV. Bd. 1902. S. 7.

5) Panse, Bericht über die Tätigkeit der Kgl. Universitäts-Ohrenklinik zu Halle a. S. vom 1. April 1890 bis 31. Mărz 1891. Dies. Arch. XXXIII. Bd. 1892. S. 38 . 
Makroskopiseh wurde Eiter im Saceus endolymphaticus festgestellt in geringer Menge von Leutert') (Fall 9), in größere Menge zum Teil mit Auftreibung des Sackes bis zu Bohnengröße von Jansen ${ }^{2}$ ), Friedrich (l. e. Fall 19) und Klug (l. e.). Bei diesem letzten Falle waren auch durch die Operation große Knochenpartien an der Hinterseite des Felsenbeins zerstört, so dah mir der makroskopische Sektionsbefund nicht eindeutig zu sein scheint.

Freien Eiter im Saceus konnten Grunert und Dallmann ${ }^{3}$ ) (Fall 2 der Todesfälle) zwar makroskopisch nicht feststellen, sie fanden den Saccus aber verdickt und mißfarben und nehmen an, daf durch seine Infektion vom Labyrinth aus der Kleinhirnabszeb entstanden sei.

Wenn Schulzed) von seinem Fall sagt, dab das Empyem durch die Sektion einwandsfrei festgestellt wurde, so möchte ich dem bei der fehlenden mikroskopischen Untersuchung nicht beistimmen. Es ist mir auch nicht klar, wie der kirsehgroße Sack prall mit Eiter gefüllt sein konnte, während er doch an zwei Stellen eingerissen war; ferner wie die Diagnose auf Plattenepithelauskleidung dieses großen Abszesses möglich war, selbst wenn eine mikroskopische Untersuchung vorgenommen wäre, von der aber nichts erwähnt ist. Es scheint mir gänzlich ausgeschlossen, daß dies feine Epithel in einem solchen Eiterherde sich sollte erhalten haben. Ich erwähne dies ausdruicklich, weil in keinem aller für unsere Frage hier in Betracht kommenden Fälle mikroskopische Einzelheiten am Saccus, die gerade hier sehr wünschenswert wären, beschrieben sind.

Bei dem einen (Fall 2) der von Jobson Horne ${ }^{5}$ ) beschriebenen Fälle kommunizierte ein Abszeß, der in der Gegend des S. 217.

1) Leutert, Über die otitische Pyămie. Dieses Arch. XLI. Bd. 1896.

2) Jansen, Zur Kenntnis der durch Labyrintheiterung inducierten, tiefen extraduralen Abszesse in der binteren Schädelgrube. Dieses Archiv. XXXV. Bd. 1893. S. 290.

3) Grunert und Dallmann, Jahresbericht der Kgl. UniversitätsOhrenklinik zu Halle a. S. vom 1. April 1904 bis 31. März 1905. Dieses Arch. LXV. Bd. 1905. S. 55.

4) Schulze, Zur Kenntnis des Empyems des Saccus endolymphaticus. Dieses Archiv. LVII. Bd. 1903. S. 67.

5) Jobs on Horne, The formation of a circumscribed intradural abscess at the site of the saccus endolymphaticus. The journal of laryngology, rhinology, and otology. 1900. 
Saccus zwischen den beiden Durablättern gesessen haben soll, mit der hinteren Schädelgrube, bei dem anderen (Fall 1) zeigte der Saccusabszeb zwei Perforationen, eine in die hintere Schädelgrube und eine in den Sinus, wo hierdurch eine septische Thrombose entstanden war.

Der letzte Fall liegt ähnlich verwickelt, wie der von Grunert und Dallmann (1. c. Fall 5), bei dem das Empyem des Saccus endolymphaticus durch eine kleine Fistel mit einem Extraduralabszeb an der Hinterseite des Felsenbeins in Verbindung: stand, der wieder zur Bildung eines Kleinhirnabszesses gefübrt hatte.

Einen Fall von $G \mathfrak{u l l}^{1}$ ) habe ich leider nicht im Original nachlesen können; ich darf mich hier auf die Notizen von Boeseh stützen, die folgendermaßen lauten: "In rechter Kleinhirnhälfte ein oberflächlicher Abszeß, etwa 6 Unzen grünlichen, nicht stinkenden Eiter enthaltend. Die oberflächliche Wand des Abszesses war der Dura an einer kleinen Stelle adhärent, welche dem Eintritt des Aquaeductus vestibuli entspricht". Aus dieser Beschreibung kann man, glaube ich, nicht den Schluß ziehen, daß es auf dem Wege des Aquaeductus vestibuli zu einem Empyem des Saccus endolymphatious gekommen ist.

In zahlreichen Sektionsberichten wird anseheinend ein besonders großer Wert darauf gelegt, daß der Abszeß ,in der Gegend des Saceus endolymphaticus" gesessen habe, und es wird hauptsächlich auf diesen Befund hin die Diagnose auf Saccusempyem gestellt. Meiner Ansicht nach mit Unrecht, da.duroh den Sitz an dieser Stelle noeh nichts bewiesen ist. Wie man sich an einem Präparate, an dem der Sinus bis in den Bulbus hinauf mit Fortnahme der diesen deckenden hinteren Knochenwand jederzeit leicht überzeugen kann, muß jeder einigermaßen große Abszeß an der Hinterseite des Felsenbeins, natürlich mit Ausnahme der Gegend des Porus acusticus internus "in der Gegend des Saccus endolymphaticus" sitzen. Hier ist wirklich Raummangel vorhanden. Mein oben mitgeteilter Fall zeigt, wie sehr man sich in dieser Beziehung bei nur makroskopischer Betrachtung täuschen kann.

Auch über den Zustand des Knochens am Boden eines solchen Abszesses kann man sichern Aufschluß nur durch die mikroskopische Untersnchung gewinnen.

1) Gull, Guys Hospital reports. III. Series. Vol. III. 
Von Leutert (l. e.) wird noch auf eine andere Mögliohkeit der Entstehung eines Saceusempyems hingewiesen, die bei mehreren der aufgezählten Fälle wohl hätte in Betracht gezogen werden können, nämlich so, daß der Aquaeductus vestibuli auf seinem Verlaufe von der Knochencaries ergriffen wird und nun in seinem letzten, vielleicht sehr kurzen Endstiuck, die Eiterung ins Schädelinnere bringt, ohne direkt vom Labyrinth her den Leitungsweg gebildet zu haben. Diese Erklärung ist sicher nicht von der Hand zu weisen und für einige der beschriebenen Fälle möglich, wo so ausgedehnte Zerstörungen des Knochens in der Gegend des Aquaeductus vestibuli vorhanden waren. Etwas weiter gehen in dieser Beziehung noch $\mathrm{Kämmel} \mathrm{(l.} \mathrm{c.)} \mathrm{und} \mathrm{Muck}$ (1. c.), die, wie schon oben erwähnt, für ihre Fälle einen direkten Durchbruch der Knocheneiterung in den Saccus annehmen.

Auf einen anderen Punkt möchte ich noch aufmerksam machen, der bei nur makroskopischer, aber auch noch bei mikroskopischer Untersuchnng zu Täuschungen Veranlassung geben kann. Es ist dies die Aufsplitterung der Dura am Rande eines extraduralen Abszesses. Man kann wahrlich leicht zu dem Glauben kommen, hier die Randteile der beiden Blätter vor sich zu haben, die den Sacous endolymphaticus einschließen.

DaB Eiter aus dem Labyrinth durch den Aquaeductus vestibuli in den Sacous wandern kann, mub man nach der Beobachtung von Politzer (l. c.) annehmen. Der Erklärung aber, wie ein bohnen- resp. kirschgroßer Abszeß auf diese Weise entstehen kann, setzen sich doch erhebliche Sehwierigkeiten entgegen. Das darf man doch wohl rom allgemein pathologischen Standpunkte aus annehmen, - und Boesch hat diese Punkte anch berührt - daß beim Einbruche einer Eiterung in das Labyrinth, die sich meist verhältnismäBig langsam vollziehen wird, oder auch schon vorher, eine Schwellung der Labyrintbgebilde, also auch der Epithelien des Aquaeductus vestibuli eintreten wird. Dadurch wird dieser ohnehin schon sehr enge Gang, der nach Schwalbe ${ }^{1}$ ) bis $0,25 \mathrm{~mm}$ messen $\mathrm{kann}$, noch mehr verengt, so dab er für die Passage von Eiterkörperchen nur sebr wenig Raum bieten dürfte. Wie es da durch ein so feines Röhrohen, in dem doch keine eigentliche Strömung nach der Dura hin herrscht, zu einem großen Abszeb im Saceus kommen soll, der unter erheblichen Drucke steht, ist nicht leicht zu erklären. Mög-

1) Schwalbe, Lehrbuch der Anatomie der Sinnesorgane. 1887. S. 321. 
Kritische Bemerkungen über das Empyem des Saccus endolymph. usw. 285

lich wäre es ja, dab aus der feinen Vena aquaeductus vestibuli, die nach Siebenmann neben dem eigentliohen Aquaeductus in einer besonderen Rinne verläuft, viele Leukocyten ausgeschieden werden in den Saccus, der durch den Ductus vestibuli infektiöses Material erhalten hat.

Mit der Deutung von Eiter im Aquaeductus vestibuli oder auch cochleae wird man aber auch bei mikroskopischer Untersuchung sehr vorsichtig sein müssen, da doch in verhältnismäßig dicken Schnitten, die einen langen Entkalkungsprozeß durehgemacht haben, die Entscheidung - ob gequollene Epithelien oder Leukocyten - oft unmöglich sein wird.

Wie aus diesen Ausführungen zu ersehen ist, mub die Frage nach der Entstehung des Saccusempyems und der Bedeutung des Aquaeductus vestibuli als Infektionsweg noch fast unbeantwortet gelassen werden; denn sehr viele der in der Literatur beschriebenen Fälle halten einer Kritik nicht stand. Anch hier gilt, was $\mathrm{Hinsberg}{ }^{2}$ ) ebenso in anderen Fragen der Labyrintheiterungen als Forderung aufstellt - noch einmal ganz von vorne anzufangen. Nur zahlreiche, mühsame mikroskopische Untersuchungen vermögen hier eine Klärung zu bringen.

1) Siebenmann, Mittelohr und Labyrinth. v. Bardelebens Handbuch. V. Bd. 2. Abteilung. S. 319.

2) Hinsberg, Über Labyrintheiterungen. Verhandl. der Deutschen otolog. Gesellschaft 1906. S. 50. 